\title{
Connections beween photospheric current helicity, flares and solar subsurface kinetic helicity
}

\author{
Yu Gao ${ }^{1}$, Junwei Zhao ${ }^{2}$, and Hongqi Zhang ${ }^{1}$ \\ ${ }^{1}$ Key Laboratory of Solar Activity, National Astronomical Observatories, \\ Chinese Academy of Sciences, Beijing, P. R. China \\ email: gy@bao.ac.cn \\ ${ }^{2}$ W.W.Hansen Experimental Physics Laboratory, \\ Stanford University, Stanford, CA94305-4085, U.S.A.
}

The $\alpha$-effect is central to magnetic field generation in $\alpha \Omega$ dynamo theory (Parker, 1955), however, it lacked observational and experimental supports over a long term (Sokoloff, 2007). Mirror asymmetries of magnetic field (magnetic helicity) were not observed until the recent 20 years (Seehafer, 1990; Pevtsov et al., 1995, Bao \& Zhang, 1998). Later, the advancement in local helioseismology has made possible the measurements of solar subsurface velocity field and the vorticity (or kinetic helicity) as well (Zhao \& Kosovichev, 2003). Apparently, frozen flow with the magnetic field in the fluid of high magnetic Reynold number is a necessary condition for the $\alpha$-effect in the framework of Parker's dynamo model.

In this paper, we briefly introduce some of our recent works related to the studies of solar subsurface flows and their connections with photospheric properties.

Comparison between subsurface kinetic helicity and photospheric current helicity in different active regions has been performed. A statistical study by Zhao (2004) showed that the subsurface kinetic helicity inside active regions observed by the Solar Heliospheric Observatory / Michelson Doppler Imager (SOHO/MDI) seemed to have a hemispheric preponderance, like what magnetic (or current) helicity observations had shown. Gao, Zhang, \& Zhao (2009) analyzed the connection between the photospheric current helicity, calculated from vector magnetograms observed by Huairou Solar Observing Station, and the subsurface kinetic helicity measured from MDI observations in 38 solar active regions. Similar analysis was performed by Maurya et al. (2011) as well.

High cadence simultaneous observations of vector magnetic field (Hoeksema et al. 2012) and subsurface flow field by SDO/HMI (Zhao et al. 2012) make possible the comparison between two kinds of helicities during the evolution of an active region. We studied 5-day observations of two active regions, and found that the variations of kinetic and current helicities exhibit good correlations, and this might be a good manifestation of magnetic field and plasma frozen together. Although the speculation of the depth of $\alpha$-effect is still under debate, it is expected that some relevant characteristic might be captured even near the photosphere. Two flare-productive active regions NOAA AR 11158 and AR 11283 have been studied. For the weighted helicities, we find that the correlation of evolutional curves of two kinds of helicity are larger than 0.60 . It is very interesting to see the weighted helicities attain a high correlation during the evolution of both active regions, in particular when considering that these two helicities are calculated from very different data. Another fact we cannot ignore is that despite the high positive correlation in the evolutionary curves of the two helicities, the signs of the two helicities do not often stay the same. In particular, for AR 11158, the signs of the two helicities are more 
often opposite than same. Therefore, although the evolution of the two helicities is in positive correlation, it is quite likely that we get a negative or no correlation if we pick just one snapshot randomly. This may help explain why our previous study did not find any correlation between two kinds of helicities (Gao, Zhang, \& Zhao, 2009). The results presented in this paper also give us some guidance of how a statistical study with many active regions should be carried out. Additionally, we also note the independent physical meaning of the previoius study, where the result manifest the correlation of magnitudes and signs of helicities in different active regions in comparison to here it manifests the correlation of the variance of two helicities during the evolution of active region.

It is of great interest to investigate the connections between solar subsurface dynamics and solar activities. Zhao \& Kosovichev (2004) found that the residual vorticity peaks in the activity belts and is of opposite sign in each hemisphere from a time-distance analysis of MDI data. Then a series of studies have been performed (Komm et al. 2004a, b; 2005; Mason et al. 2006; Reinard et al. 2010) and a proposed parameter can even provide advance notice of flare occurrence as it increased 2 to 3 days before the flare.

The subsurface velocities from HMI time-distance data-analysis pipeline (Zhao et al. 2012) are used to study the evolution of subsurface kinetic helicity in a few flareproductive active regions for the purpose to identifying possible connections between subsurface dynamics and major solar flares. Our analysis shows that for almost all studied major flares, some bumps in subsurface kinetic helicity occurred near the time of flare occurrence. This prompts a better understanding of how subsurface dynamics can lead to coronal activities.

SDO is a NASA mission, and HMI project is supported by NASA contract NAS5-02139 to Stanford University. This work is partially supported by the National Natural Science Foundation of China under grants 11028307, 10921303, 11103037, 11173033, 41174153 and Chinese Academy of Sciences under grant KJCX2-EW-T07.

\section{References}

Bao, S. D. \& Zhang, H. Q. 1998, ApJ., 496, L43

Gao, Y., Zhang, H., \& Zhao, J. 2009, MNRAS., 394, L79

Hoeksema, J. T., Liu, Y., \& Hayashi, K. 2012, Sol. Phys., in press

Komm, R. W., Corbard, T., Durney, B. R., Gonzalez Hernandez, I., Hill, F., Howe, R., \& Toner, C. $2004 \mathrm{a}$, ApJ., 605, 554

Komm, R. W.,Howe, R.,Gonzalez Hernandez, I., Hill, F., Haber, D., Hindman, B., \& Corbard, T. 2004b, in SOHO 14 Helio- and Asteroseismology: Towards a Golden Future, ed. D. Danesy (ESA SP-559; Noordwijk: ESA), 520

Komm R., Howe R., Hill F., Gonzalez Hernandez I., \& Toner C. 2005, ApJ, 630, 1184

Mason, D., Komm, R., Hill, F., Howe, R., Haber, D., \& Hindman, B. 2006, ApJ, 645, 1543

Maurya, R. A., Ambastha, A., \& Reddy, V. 2011, J. Phys. Conf. Ser., 271, 012003

Parker, E. 1955, ApJ, 122, 293

Pevtsov, A. A., Canfield, R. C., \& Metcalf, T. R. 1995, ApJ, 440, L109

Reinard, A. A., Henthorn, J., Komm, R., \& Hill, F. 2010, ApJ., 710, L121

Seehafer N. 1990, SoPh, 125, 219

Sokoloff, D. 2007, Plasma Phys. Control. Fusion, 49, 447

Zhao, J., Couvidat, S., Bogart, R. S., Parchevsky K. V., Birch, A. C., Duvall, T. L., Jr., Beck J. G., Kosovichev, A. G., \& Scherrer, P. H. 2012, SoPh, 275, 375

Zhao, J. \& Kosovichev, A. G. 2003, ApJ., 591, 446

Zhao, J. \& Kosovichev, A. G. 2004, ApJ., 603, 776

Zhao, J. 2004, Chap. 5, PhD Thesis, Stanford Univ. 